\title{
$\sigma$-meson: Four-quark versus two-quark components and decay width in a Bethe-Salpeter approach
}

\author{
Nico Santowsky $\odot,{ }^{1, *}$ Gernot Eichmann $\odot,{ }^{2,3, \dagger}$ Christian S. Fischer $\odot,{ }^{1,4, \hbar}$ Paul C. Wallbott, ${ }^{1}$ and Richard Williams ${ }^{1}$ \\ ${ }^{1}$ Institut für Theoretische Physik, Justus-Liebig Universität Gießen, 35392 Gießen, Germany \\ ${ }^{2}$ LIP Lisboa, Av. Prof. Gama Pinto 2, 1649-003 Lisboa, Portugal \\ ${ }^{3}$ Departamento de Física, Instituto Superior Técnico, 1049-001 Lisboa, Portugal \\ ${ }^{4}$ Helmholtz Forschungsakademie Hessen für FAIR (HFHF), GSI Helmholtzzentrum \\ für Schwerionenforschung, Campus Gießen, 35392 Gießen, Germany
}

(Received 20 July 2020; accepted 26 August 2020; published 11 September 2020)

\begin{abstract}
We study the dynamical generation of resonances in isospin singlet channels with mixing between twoand four-quark states. To this end, we generalize a Bethe-Salpeter approach to four-quark states employed previously to accommodate for mixing diagrams. The $q \bar{q} q \bar{q}$ and $q \bar{q}$ components of the Bethe-Salpeter wave function (with light quarks $q \in\{u, d\}$ ) are determined consistently in a symmetry-preserving truncation of the underlying Dyson-Schwinger equations. As a prominent example, we deal with the isospin-singlet $0^{++}$meson with light quark content. We find that the $\pi \pi$ contribution of the four-quark component is mainly responsible for the low (real part of the) mass of the resulting state. We also study the analytic structure in the complex momentum plane and find a branch cut at the two-pion threshold and a singularity in the second Riemann sheet indicating a considerable decay width. Our findings are in excellent qualitative agreement with the general picture for the $\sigma / f_{0}(500)$ that emerged in the past two decades from dispersive approaches.
\end{abstract}

DOI: $10.1103 /$ PhysRevD.102.056014

\section{INTRODUCTION}

There is perhaps no other state in the low-energy spectrum of QCD that has been puzzled over so intensely over the past decades than the isoscalar-scalar meson, the $f_{0}(500)$ or $\sigma$ meson. It plays a very important role in our understanding of chiral symmetry breaking, it is one of the defining quantities in model building and, together with its flavor partners in the light scalar nonet, it has properties that do not go easily together with a conventional mesonic $q \bar{q}$ picture of its internal structure. Once discarded from the particle data book, the light scalar meson nonet has been reintroduced again at the end of the past century. Only in the 2012 edition, the $\sigma$ meson has been renamed $f_{0}(500)$ and accepted with much smaller uncertainties in its mass and width as ever before, mainly due to progress in the dispersive analysis of experimental data; see [1-4] and Refs. therein. Many aspects of this fascinating story

\footnotetext{
*nico.santowsky@theo.physik.uni-giessen.de 'gernot.eichmann@tecnico.ulisboa.pt

*christian.fischer@theo.physik.uni-giessen.de
}

Published by the American Physical Society under the terms of the Creative Commons Attribution 4.0 International license. Further distribution of this work must maintain attribution to the author(s) and the published article's title, journal citation, and DOI. Funded by SCOAP. together with a detailed discussion on the theoretical background and the intricacies of data analysis can be found in the review article Ref. [5].

The notion that the multiplet of light scalar mesons is incompatible with a conventional $q \bar{q}$ picture goes back some way [6]: by assuming a dominant four-quark structure, interesting properties like inverted mass hierarchies and decay patterns are naturally explained. This picture is supported by effective theory studies and large- $N_{c}$ arguments (see, e.g., [5,7-12] and references therein) as well as lattice calculations [13-16] and functional methods $[17,18]$.

In principle, an experimentally observed state may be a mixture of several states with different internal structure but the same quantum numbers. Thus, it is by no means clear whether the $f_{0}(500)$ is indeed a pure four-quark state or has some overlap with the corresponding conventional $q \bar{q}$ state, thus generating subdominant $q \bar{q}$ contributions to its composition. This idea has been elaborated upon in a number of works, including studies within linear sigma models $[8,19,20]$, instanton induced mixings in tetraquark models [21], unitarized quark model calculations [22] and unitarized chiral perturbation theory, and leading $1 / N_{c^{-}}$ considerations [23-25]. Evidence from these studies clearly points towards a small but significant admixture of $q \bar{q}$ components to the four-quark state. Note that a similar situation also appears in the heavy-quark sector, 
where several XYZ states are discussed to be dominated by four-quark components with a heavy and a light quarkantiquark pair. However, at least for the isospin-singlet cases like the $\chi_{c 1}(3872)$, substantial admixtures of $\bar{c} c$ components seem possible [26].

In this work, we address this question on the example of the isospin-singlet $0^{++}$meson with light quark content using the functional approach via Dyson-Schwinger (DSE) and Bethe-Salpeter equations (BSE). In previous work within this approach, the state in question has been treated either as a conventional $q \bar{q}$ state using a range of truncations for the quark-gluon interaction, or as a pure four-body $q \bar{q} q \bar{q}$ state. Using the rainbow-ladder approach in the $q \bar{q}$ BSE, this state is found at a (real) mass of the order of $650 \mathrm{MeV}$ [27]. In various beyond rainbow-ladder calculations with sophisticated interactions, its (real) mass is shifted to (much) larger values beyond $1 \mathrm{GeV}$ [28-30]. This does not support a large quark-antiquark contribution to the $f_{0}(500)$. On the other hand, the treatment of the lightest scalar meson as a four-quark state, once in the four-body approach using a Faddeev-Yakubovsky equation [18] and once in a reduced two-body approach [17] using internal meson and diquark degrees of freedom, led to substantially smaller masses around $400 \mathrm{MeV}$. Moreover, it turned out that the latter two approaches both favored a leading internal $\pi \pi$ component in the state's wave function, thus suggesting the identification with the $f_{0}(500)$.

While these studies are already suggestive, they can be improved in two respects. First, it needs to be studied whether the picture of $\pi \pi$ dominance still persists when the coupled system of Bethe-Salpeter equations for the fourquark $q \bar{q} q \bar{q}$ and conventional $q \bar{q}$ states is considered. Second, previous extractions of the $\sigma$ mass in the fourquark formalism focused on the real part only and were not able to determine the width of the state in question. We will address both problems in this work.

The paper is organized as follows: we first detail our derivation of the coupled system of BSEs for a state with four-quark $q \bar{q} q \bar{q}$ and two-quark $q \bar{q}$ components in Sec. II. To keep matters as simple as possible, we use the twobody approximation of the four-body equation introduced in [17]. In Sec. III A, we then detail our model for the underlying quark-gluon interaction and discuss briefly the corresponding DSE for the quark propagator. Together, the resulting quark and the interaction provide a self-consistent input into the various BSEs studied in this work. In Sec. III B, we summarize an approach to deal with the analytic structure generated by the two-pion cut, developed in Ref. [31] and adapted here for the case of the $\sigma$ meson. Section IV is devoted to a discussion of our results. In Sec. IV A, we discuss our results for the coupled system of four- and two-quark BSEs and study the impact of mixing effects. In Sec. IV B, we then determine the decay width of the pure two-quark component. We summarize and conclude in Sec. V.

\section{THE FUNCTIONAL APPROACH}

\section{A. The four-body Bethe-Salpeter equation}

The equations of motion for $n$-quark bound states, the Bethe-Salpeter equations (BSEs) are derived from the (2n)quark scattering matrix $T^{(n)}$ and the corresponding scattering kernel $K^{(n)}$,

$$
T^{(n)}=K^{(n)}+K^{(n)} G_{0}^{(n)} T^{(n)} .
$$

Here, $G_{0}^{(n)}$ denotes the product of $n$ dressed quark propagators. By defining a bound-state amplitude (BSA) $\Gamma^{(n)}$, which carries the Dirac, color, and flavor structure of the bound state in question, the $T$ matrix at the physical pole $P^{2} \rightarrow-M^{2}$ of the bound state propagator $D$ is given by

$$
T^{(n)} \stackrel{P^{2}}{\stackrel{-}{\approx} M^{2}} \frac{\Gamma \bar{\Gamma}}{P^{2}+M^{2}} .
$$

Inserting this expression into Eq. (1) leads to the homogeneous on shell $n$-quark BSE,

$$
\Gamma^{(n)}=K^{(n)} G_{0}^{(n)} \Gamma^{(n)} .
$$

In this paper, we focus on two-quark and four-quark bound states, and we further denote $\Gamma^{(2)} \equiv \Gamma$ as a two-quark BSA and $\Gamma^{(4)} \equiv \Psi$ as a four-quark BSA.

The complete scattering kernel $K^{(4)}$ occurring in the four-body BSE,

$$
\begin{gathered}
\Psi=K^{(4)} G_{0}^{(4)} \Psi, \\
K^{(4)}=\tilde{K}^{(2)}+\tilde{K}^{(3)}+\tilde{K}^{(4)}
\end{gathered}
$$

can be decomposed in three contributions $\tilde{K}^{(2,3,4)}$ containing irreducible two-, three- and four-quark interactions [32]. In the following, we adopt the strategy of Refs. [17,18] and neglect the latter two; i.e., we set $\tilde{K}^{(3)}=\tilde{K}^{(4)}=0$. The basic idea behind this approximation is the notion that the two-body interactions may well dominate the state in question. This is motivated by two considerations: first, the broad decay width of the experimental $f_{0}(500)$ into two pions suggests a dominance of internal two-body correlations. Conversely, if strong quarkantiquark correlations inside the four-quark state form a two-pion internal structure, its singularity structure naturally has a strong effect on the BSE that almost inevitably will dominate the equation and dwarf contributions from $\tilde{K}^{(3)}$ and $\tilde{K}^{(4)}$. Second, a similar approximation has been applied with great success in the baryon sector. There, strong two-body correlations naturally lead to a diquarkquark picture, which in turn leads to a spectrum in one-toone agreement with experiment; see, e.g., [33,34] and references therein. Moreover, for baryons it can be shown 
explicitly that the leading part of the irreducible three-body interaction (in terms of a skeleton expansion) is small [35]. While all these arguments are not strictly rigorous, they provide plausible justification for neglecting the irreducible many-body interactions $\tilde{K}^{(3,4)}$ also in the four-quark case. Practicability arguments complement these considerations.

The contribution $\tilde{K}^{(2)}$ containing all irreducible twobody interactions inside the four-quark state contains various incarnations of the two-body scattering kernel $K_{i j}^{(2)}$ between two quarks $i$ and $j$,

$$
\begin{aligned}
\tilde{K}^{(2)} & =\underbrace{K_{12}^{(2)} S_{3}^{-1} S_{4}^{-1}+K_{34}^{(2)} S_{1}^{-1} S_{2}^{-1}-K_{12}^{(2)} K_{34}^{(2)}}_{=: \tilde{K}_{(12)(34)}^{(2)}}+\text { perm. } \\
& =\sum_{a} \tilde{K}_{a}^{(2)} .
\end{aligned}
$$

Explicit indices 1,2,3,4 denote the four (anti)quarks as ingredients of the four-quark bound state and the summation over $a$ picks up the three possible combinations (12) (34),(13)(24),(14)(23) of two-body interactions.

\section{B. The two-body Bethe-Salpeter equation}

In order to be able to extract a two-body Bethe-Salpeter equation for the four-quark $q \bar{q} q \bar{q}$ state, we slightly reformulate the problem [17]. First, we define a four-body $\mathrm{T}$ matrix $T_{a}$ that is generated by $\tilde{K}_{a}^{(2)}$,

$$
T_{a}=\tilde{K}_{a}^{(2)}+\tilde{K}_{a}^{(2)} G_{0}^{(4)} T_{a}=\tilde{K}_{a}^{(2)}+T_{a} G_{0}^{(4)} \tilde{K}_{a}^{(2)} .
$$

Furthermore, we note that the BSA, Eq. (4), can be split into three separate parts by inserting Eq. (6),

$$
\Psi=\sum_{a} \tilde{K}_{a}^{(2)} G_{0}^{(4)} \Psi:=\sum_{a} \Psi_{a} .
$$

Acting with $T_{a} G_{0}^{(4)}$ onto $\Psi$ and using (7), one then obtains

$$
\Psi_{a}=T_{a} G_{0}^{(4)}\left(\Psi-\Psi_{a}\right)=\sum_{b \neq a} T_{a} G_{0}^{(4)} \Psi_{b}
$$

which is still an exact four-body equation apart from neglecting the kernels $\tilde{K}^{(3)}$ and $\tilde{K}^{(4)}$.

Since the T matrices $T_{a}$ contain effects from two-body interactions in the same combination of (anti)quark legs only, they are prone to develop singularities in the respective channels, with the quantum numbers of mesons and (anti)diquarks. The two-body approximation of the fourbody equation then amounts to replacing $T_{a}$ with a pole ansatz analogously to Eq. (2). Assuming that the spinmomentum structure of the Bethe-Salpeter amplitudes factorizes, the full amplitude $\Psi$ can then be decomposed into meson-meson and diquark-antidiquark substructures $\Phi_{a}$. We thus obtain
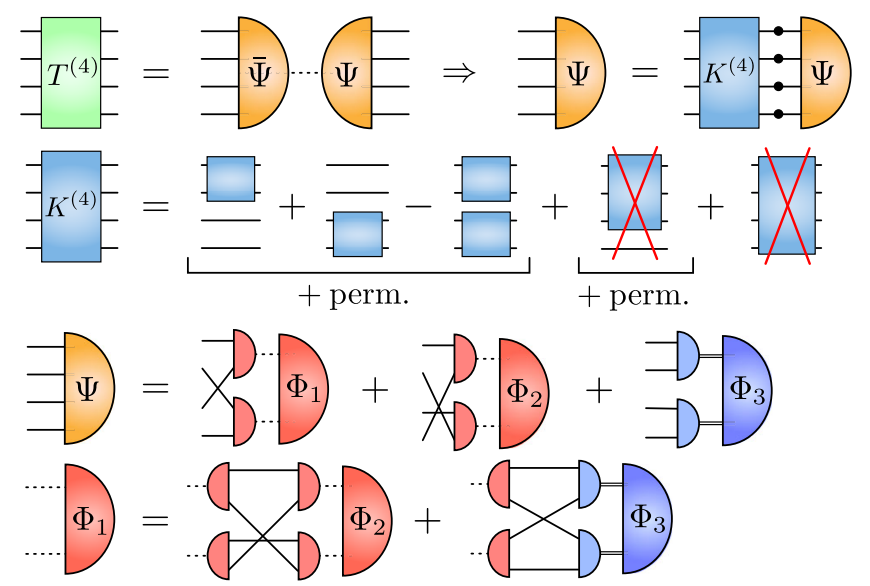

FIG. 1. Diagrammatic representation of the basic quantities used in deriving the pure two-body/four-quark BSE. In the first line, we display the representation of the bound state (2) together with its corresponding BSE (3). In the second line, we give the explicit decomposition of the interaction kernel $K^{(4)}$ in terms of irreducible two-, three- and four-body interactions. The red crosses indicate truncations, justified and explained in the main text. The third line displays the reduction of the four-body amplitude into a sum of two-body amplitudes featuring internal mesons (dashed lines) and (anti)diquarks (double lines). One of the resulting effective two-body equations is given in the lowest line. The other two equations are obtained under permutations in the index set $\{1,2,3\}$, thus spanning the whole coupled system of two-body BSEs (for equal quark masses $\Phi_{1}$ and $\Phi_{2}$ are identical).

$$
\Psi_{a}=\left(\Gamma_{12} \otimes \Gamma_{34}\right) G_{0}^{(2,2)} \Phi_{a}
$$

for $a=(12)(34)$ and similar expressions for the other combinations. Here, $G_{0}^{(2,2)}$ is a combination of two meson propagators or a diquark and an antidiquark propagator, respectively, and $\Gamma_{i j}$ are the corresponding two-body Bethe-Salpeter amplitudes. The representation Eq. (10) is in some sense a "physical basis" in that it builds a representation of $\Psi_{a}$ in terms of reduced internal Dirac, flavor, and color structure from a physical picture. The algebraic structure of the tetraquark-meson and tetraquarkdiquark vertices $\Phi_{a}$ depend on the respective quantum numbers of the investigated four-quark state. For scalar four-quark states and (pseudo)scalar ingredients, e.g., those amplitudes are flavor and color singlets and Lorentz scalars.

With Eq. (10), we effectively solve for the vertices $\Phi_{a}$ while making use of solutions of the two-quark BSE for the amplitudes $\Gamma_{i j}$. The interaction kernel elements for the internal vertices $\Phi_{a}$ are quark exchange diagrams as visualized in the last line of Fig. 1.

\section{Inclusion of a two-quark component}

We now extend the truncation for the four-body equation (9) by adding a phenomenologically motivated 
two-quark component into the Bethe-Salpeter amplitude. For $a=(12)(34)$ in Eq. (10), this amounts to

$$
\Psi_{a}=\left(\Gamma_{12} \otimes \Gamma_{34}\right) G_{0}^{(2,2)} \Phi_{a}+K_{13}^{(2)} K_{24}^{(2)} S_{34} G_{0}^{(2)} \Gamma_{12}^{*}+\text { perm. },
$$

where $\Gamma_{12}^{*}$ is the Bethe-Salpeter amplitude of a quarkonium state with the same quantum numbers as the four-body state connected to the propagator lines 1 and 2, and the quark propagator $S_{34}$ connects the lines 3 and 4 . This is equivalent to extending the physical basis discussed above with another possible basis element. Note that these extensions only appear in those $\Psi_{a}$ with two-body interactions between quark-antiquark pairs, i.e., meson-meson contributions. They do not appear in the $\Psi_{a}$ with two-body interactions between quark-quark pairs, i.e., in diquark/ antidiquark contributions.

The corresponding two-quark $T$ matrix that contains this quarkonium state is determined by [cf. Eq. (1) with $n=2$ ],

$$
T^{(2)}=\left(\mathbb{1}-K^{(2)} G_{0}^{(2)}\right)^{-1} K^{(2)} .
$$

The corresponding four-quark $T$ matrix that contains the four-body component added in Eq. (11) is denoted by

$$
T_{a}^{(4,2)}=K_{13}^{(2)} K_{24}^{(2)} S_{34} G_{0}^{(2)} T_{12}^{(2)} G_{0}^{(2)} S_{34} K_{24}^{(2)} K_{13}^{(2)} .
$$

As a result, the master equation (9) then contains the twobody equation for $\Gamma^{*}$ as an additional element, and the equations for the four-body meson-meson and diquarkantidiquark components of the full BSA are modified by additional terms containing $\Gamma^{*}$. The resulting system of equations is shown diagrammatically in Fig. 2. In its derivation, we have frequently used the BSE,

$$
\Gamma^{(2)}=K^{(2)} G_{0}^{(2)} \Gamma^{(2)},
$$

for the internal meson and diquark states in order to absorb two-body interaction kernels in the corresponding BetheSalpeter amplitudes. We observe $q \bar{q}$ contributions in both BSEs for the meson-meson and diquark-antidiquark components of the four-body amplitudes as well as a backcoupling of the meson-meson and diquark-antidiquark components into the $q \bar{q}$ equation.

We wish to emphasize that at the present state the structure of the coupling between the four-quark state and the two-quark state is introduced by hand. There is some formal motivation from the fact that the Dirac structure of the new elements in Eq. (11) is part of the full basis of the four-quark amplitude and thus constitutes an extension of the physically motivated basis of meson and diquark elements. Formally, however, one would probably need to derive the putative coupling of four-quark and two-quark BSEs from a framework such as the one

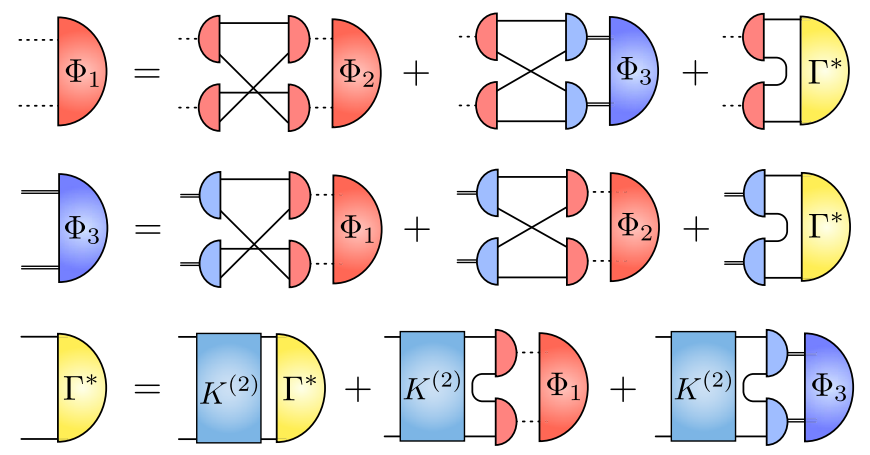

FIG. 2. The coupled system of BSEs for one of the $\pi \pi$ contributions (red; first line) and the diquark/antidiquark contribution (blue; second line) to the four-quark state as well as the coupled BSE for the quark-antiquark state (yellow; third line) with the same quantum numbers. Not shown are the additional BSE for the second $\pi \pi$ contribution $\phi_{2}$ (which is redundant in our case) and another diagram for $\Gamma^{*}$ containing $\phi_{2}$ instead of $\phi_{1}$.

given in Ref. [36]. This will be addressed in future work. Furthermore, it is interesting to observe that we would have ended up with equations of similar structure if we had used expressions such as $\Gamma_{12}^{*} S_{34}^{-1}$ for the extension of Eq. (11) much along the line of ${ }^{3} P_{0}$ models used frequently in the description of meson and baryon decays [37]. Such disconnected contributions have been considered already in Ref. [38] in the context of the original four-body equation. A potential problem with disconnected contributions is that they heavily restrict the momentum structure of the fourbody amplitude; this is avoided by our choice, Eq. (11).

\section{TECHNICAL DETAILS}

\section{A. Quark propagator, mesons, and diquarks}

The elements necessary to solve the coupled system of BSEs in Fig. 2 are dressed quark propagators, meson and diquark Bethe-Salpeter amplitudes and the corresponding propagators. All these ingredients are determined consistently from an underlying quark-gluon interaction that respects chiral symmetry. The technical details of these types of calculations have been described in many works, see, e.g., $[34,35,39]$ for reviews; thus, we only give a short summary here.

We begin by specifying the Dyson-Schwinger equation for the dressed quark propagator,

$S_{\alpha \beta}^{-1}(p)=Z_{2}\left(i \not p+m_{0}\right)_{\alpha \beta}+C_{F} \int_{q} \mathcal{K}_{\alpha \alpha^{\prime} \beta^{\prime} \beta} S_{\alpha^{\prime} \beta^{\prime}}(q)$,

with wave function renormalization constant $Z_{2}$, bare quark mass $m_{0}$ and the Casimir $C_{F}=4 / 3$ for $N_{c}=3$ from the color trace. In this form, the equation is still exact, and the interaction kernel $\mathcal{K}_{\alpha \alpha^{\prime} \beta^{\prime} \beta}$ contains the dressed gluon propagator as well as one bare and one dressed quark-gluon vertex. The Greek subscripts refer to color, flavor, and 
Dirac structure. In previous treatments of the four-quark problem $[17,18,40,41]$, the rainbow-ladder approximation has been used, and we adopt the same interaction here. Then the kernel can be written as

$$
\mathcal{K}_{\alpha \alpha^{\prime} \beta \beta^{\prime}}=Z_{2}^{2} \frac{4 \pi \alpha\left(k^{2}\right)}{k^{2}} T_{k}^{\mu \nu} \gamma_{\alpha \alpha^{\prime}}^{\mu} \gamma_{\beta \beta^{\prime}}^{\nu},
$$

with the transverse projector $T_{k}^{\mu \nu}=\delta^{\mu \nu}-k^{\mu} k^{\nu} / k^{2}$. In this formulation, both the gluon dressing function and the vector part $\sim \gamma^{\mu}$ of the quark-gluon vertex have been absorbed into an effective running coupling $\alpha\left(k^{2}\right)$, which is taken from Ref. [42] and has been discussed in detail, e.g., in [34]. This truncation guarantees the correct logarithmic behavior of the quark at large momenta. Most importantly in the present context, however, is that it allows for the preservation of the axialvector Ward-Takahashi identity by using the same interaction kernel in the Bethe-Salpeter equations for the mesons and diquarks.

With the quark propagator from Eq. (15) and the quark(anti)quark interaction kernel Eq. (16), we then solve the Bethe-Salpeter equations for light pseudoscalar mesons and scalar diquarks, which are the leading components (in terms of smallest masses) of the two-body composition of our scalar four-quark state. The explicit representation of the BSA in terms of (four) Dirac, flavor, and color components as well as details on the technical treatment of meson BSEs can be found in the review articles [34,35]. The meson/diquark propagators are then calculated via $T=\Gamma D \bar{\Gamma}$ and Eq. (1). We obtain the following masses:

$$
m_{\pi}=0.138(2) \mathrm{GeV} \quad m_{\mathrm{dq}, 0^{+}}=0.801(31) \mathrm{GeV},
$$

and the corresponding BSAs. We are working in the isospin symmetric limit $m_{u}=m_{d}$. Since the pseudoscalar meson and scalar diquark states are dominated by their leading Dirac structure, we only take those into account in BSAs appearing internally in diagrams. For the external BSA in the two-body equation of the scalar meson, we use the full structure, i.e., all four amplitudes.

With this input, we are then in a position to solve the system of coupled integral equations for the BSEs in Fig. 2. Technically, this is done as an eigenvalue problem; i.e., one introduces a general eigenvalue $\lambda$ on the left-hand side of the BSE and searches for the total momentum $P^{2}=-M^{2}$ that corresponds to $\lambda=1$. This is then the mass of the bound state/resonance in the coupled four-body/two-body system. One problem that appears in this search is the appearance of singularities in the plane of complex total momentum due to the internal meson and diquark propagators. Although the exact locations of these poles are known (from the solutions of the corresponding meson/ diquark BSEs), it remains a highly nontrivial problem to perform the integrations numerically. To avoid this problem, we determine the eigenvalue curve $\lambda\left(M^{2}\right)$ in the singularity-free region $M<2 m$, where $m$ is the mass of the lightest meson/diquark ingredient, and $M$ is the fourquark bound state mass. We then extrapolate the resulting curve further into the timelike momentum domain using rational functions. This procedure has been tested extensively for cases where one can actually do the calculation and turned out to be very stable for states with small masses such as the $\sigma$ meson studied in this work.

\section{B. Into the complex $\boldsymbol{P}^{\mathbf{2}}$ plane}

A quantitatively reliable procedure to extend the calculation of $\lambda\left(P^{2}\right)$ beyond the limitations caused by singularities in the complex momentum plane has been discussed in Ref. [31]. There, the analytic structure of the complex $P^{2}$ plane has been explored for the example of the $\rho$ meson, which has a relatively small width and is therefore well suited for a first exploration. The extension of these ideas to the four-quark BSE (or even to the coupled system of $q \bar{q} q \bar{q}-q \bar{q}$ components) is not straightforward and requires more conceptual work. For the purpose of this exploratory work, we therefore restrict ourselves to the $q \bar{q}$ component of the scalar meson and its associated BSE. The drawback of this restriction is that we cannot hope for quantitative results. As will become clear in Sec. IV A, the physical state is dominated by its four-quark components, and any restriction to the two-quark components alone will not lead to masses that should be compared with experiment. On the other hand, the technically much simpler BSE for the $q \bar{q}$ component allows us to directly study the generation of widths in a different channel than the previously studied $\rho$ meson with the potential to test the method further into the complex $P^{2}$ region of the second Riemann sheet. As will become clear in Sec. IV B, this is indeed the case and leads to qualitative insights.

We begin by a short description of the BSE for the $q \bar{q}$ component of the scalar meson that we study. Its diagrammatical representation is shown in Fig. 3. In addition to the term with effective one-gluon exchange, which arises from the rainbow-ladder construction associated with the kernel Eq. (16), we take into account a two-pion contribution that stems from corrections beyond rainbow ladder. Its origin is elucidated in detail in Refs. [31,43-45]. Note that this term is different from the corresponding one shown in Fig. 2 arising from the mixing with the four-body equation: whereas here the two-pion kernel couples directly to the $q \bar{q}$ amplitude, in the latter approach, it couples to the

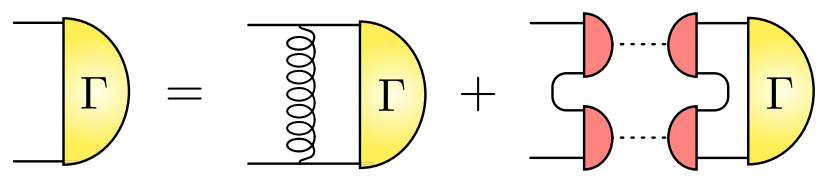

FIG. 3. The BSE for the $q \bar{q}$ component of the scalar meson. Here, the pion decay term arises not from the coupling to the fourquark equation but from contributions beyond rainbow ladder. 
four-quark amplitude. However, both terms share a crucial property: they contain two pions in the s channel, which may go on shell for certain loop momenta. As a consequence, we expect that both the mixed approach Fig. 2 and the $q \bar{q}$ approach via Fig. 3 lead to a similar structure in their respective complex plane of total momenta, namely a cut at real timelike momenta starting from $2 m_{\pi}$ and a resonance state in the second Riemann sheet. Whereas in the mixed approach we can not (yet) trace that resonance and can only extrapolate onto its real part (see the results of Sec. IVA), in the two-quark approach we have this possibility (with results discussed in Sec. IV B). Due to the similarities in structure of both approaches, we therefore expect qualitatively meaningful results from the $q \bar{q}$ approach that will carry over to the four-quark approach and can be used as guidance for future work.

We proceed by a description of the tools necessary to identify the decay width from the BSE of Fig. 3. The twopion diagram is given by

$$
\begin{aligned}
\Gamma(P, p)= & (\ldots)+C_{\pi \pi} F_{\pi \pi} \int_{l}\left[\Gamma_{1}\left(l_{+}, 2 p-l_{-}\right) S(p-l)\right. \\
& \left.\times \bar{\Gamma}_{2}\left(-l_{-}, 2 p-l_{+}\right)\right] D\left(l_{+}\right) D\left(l_{-}\right) \\
& \times \int_{q}\left[\bar{\Gamma}_{1}\left(-l_{+}, 2 q-l_{-}\right) S\left(q_{+}\right)\right. \\
& \left.\times \Gamma(P, q) S\left(q_{-}\right) \Gamma_{2}\left(l_{-}, 2 q-l_{+}\right) S(q-l)\right],
\end{aligned}
$$

with flavor and color projections already performed and the ellipsis denotes the rainbow-ladder diagram. We use the abbreviations $\int_{l}=\int \frac{d^{4} l}{(2 \pi)^{4}}, l_{ \pm}=l \pm P / 2, q_{ \pm}=q \pm P / 2$, and $S$ denotes the quark propagator, whereas $D$ stands for the pion propagator,

$$
D(p)=\left(p^{2}+m_{\pi}^{2}\right)^{-1} .
$$

In order for both integrations over $q$ and $l$ to avoid the pole locations of the pion propagators, we need to analytically deform their integration paths into the complex $l^{2}$ and $q^{2}$ planes, respectively. After angular integration, these poles generate a branch cut that is shown in the $l^{2}$ plane in Fig. 4 together with two possible choices of paths around the cuts. We have tested both choices and found them numerically equivalent.

With this path deformation, we are now in a position to determine the eigenvalue curve $\lambda\left(P^{2}\right)$ on the first Riemann sheet. Using the notation $P^{2}=(M+i \Gamma / 2)^{2}$, we display the corresponding result for the $\rho$ meson determined in Ref. [31] in Fig. 5. One clearly observes the branch cut opening at the two-pion threshold in the imaginary part of $\lambda$ (lower plane).

Unfortunately, from numerical solutions of the four-body BSE we cannot go beyond the branch cut and enter the second Riemann sheet (see Sec. IV B for a detailed

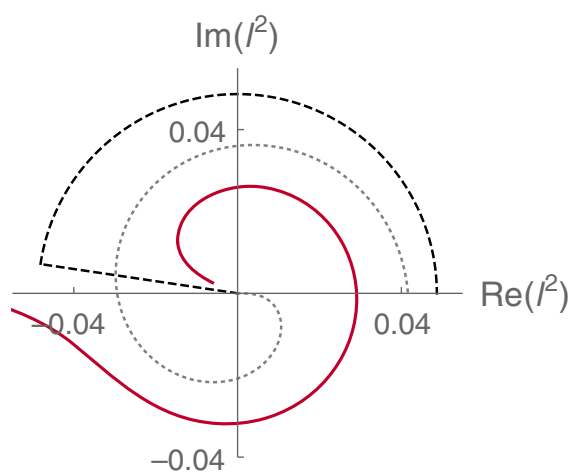

FIG. 4. We show the two-pion branch cut (red solid line) and two possible paths (dashed) avoiding the cut in the complex $l^{2}$ plane.

explanation why). Instead, this is done using a method of analytic continuation based on work by Thiele and Schlessinger and employed recently in a number of publications; see, e.g., [46-49], where details on the method can be found. In short, it amounts to using interpolating rational functions $R(x)=P(x) / Q(x)$ of degree $N$ to $N$ selected points of the function in question, thereby allowing for an analytical continuation beyond the region where the $N$ points have been taken from. One of the problems that one encounters with this method is the appearance of "fake" singularities due to imprecise cancellations in $P(x)$ and $Q(x)$. Since the location of these fake singularities and in addition also the corresponding residues (which are typically small) depend heavily on the selection of points used in the fitting procedure, they can be identified and eliminated by performing the procedure many times with random selections of points in a given region. What remains are potential "true" singularities. The variation of the location of these stable singularities within

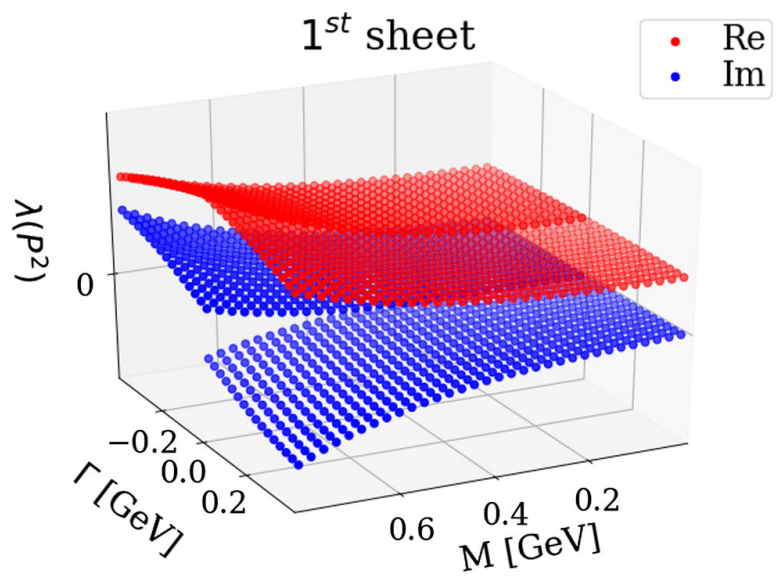

FIG. 5. Eigenvalue curves $\lambda\left((M+i \Gamma / 2)^{2}\right)$ of the BSE for the $\rho$ meson (and qualitatively similar for the $q \bar{q}$ component of the $\sigma$ meson) in the first Riemann sheet. On top is the surface for the real part of $\lambda$, below the one for the imaginary part, where a branch cut opens at the two-pion threshold. 

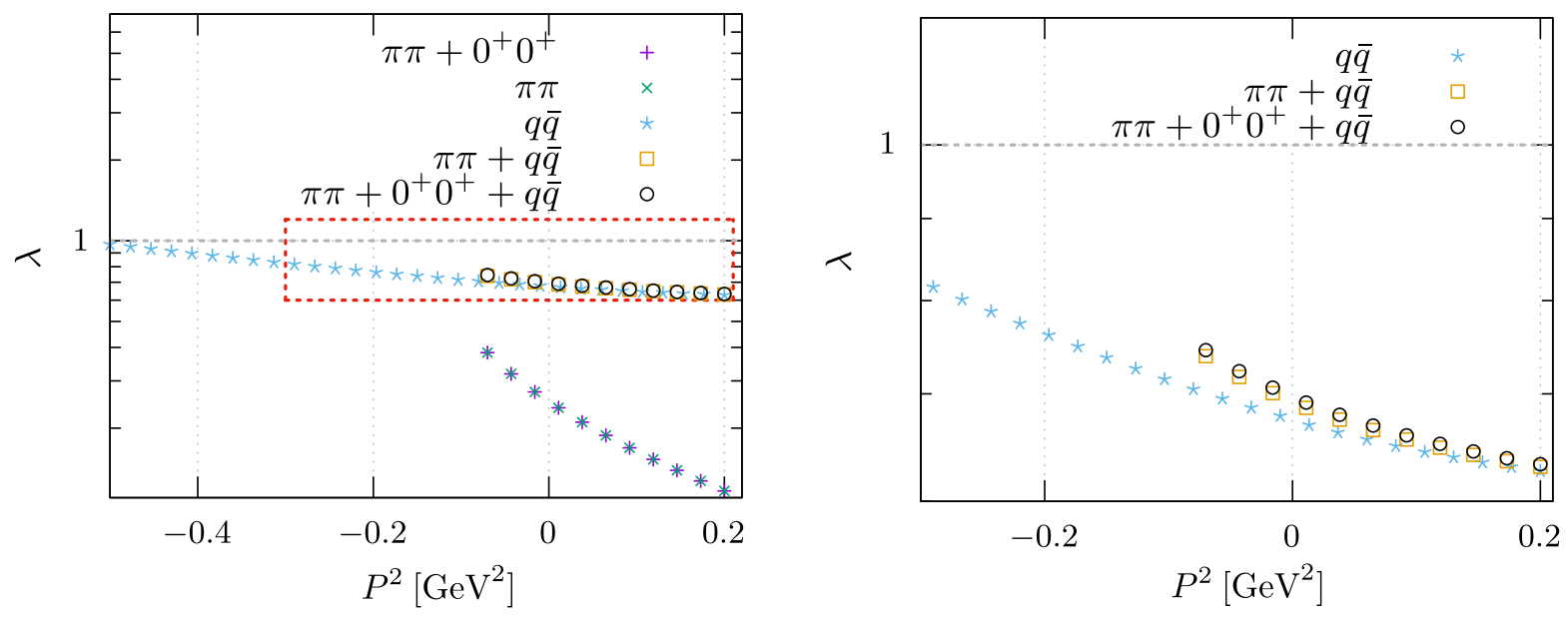

FIG. 6. The eigenvalue curves $\lambda\left(P^{2}\right)$ for different combinations of mixing ingredients. Bound states/resonances occur at $P^{2}=-M^{2}$. Shown are results of calculations with different active components in the Bethe-Salpeter wave functions; see the main text for details. The diagram on the right shows the same results as the diagram on the left but with the red area magnified.

the series of fitting procedures is quite stable and allows for a statistical error estimate. We will discuss this further in the context of our results presented in Sec. IV B.

\section{RESULTS}

\section{A. The $q \bar{q} q \bar{q}-q \bar{q}$ mixing}

Let us first come back to the mixed system of four- and two-quark states, Fig. 2. We have performed calculations for a $J^{P C}=0^{++}$state consisting of light $u$ and $d$ quarks. As internal substructures of the four-quark state, we take into account two pions for the mesonic component and two scalar diquarks for the diquark component. Thus in total, our wave function contains three components. In the practical calculation, we can switch these on and off, such that we are able to gauge the impact of each individual component. In Ref. [17], it has already been noted that the diquark component plays only a negligible role as compared to the two-pion contribution. Here, we need to study whether this is still the case once the mixing with the $q \bar{q}$ state is taken into account.

Our results for the corresponding eigenvalue curves are displayed in the two diagrams of Fig. 6. We first reproduced the results of Ref. [17]: the eigenvalue curves for the twopion contribution (green $\mathrm{x}$ ) and the "two-pion plus diquark" case (violet cross) are on top of each other. This result changes slightly in the full calculation using all three components, as can be seen by comparing the curves with two-pion plus $q \bar{q}$ (orange boxes) with the full result (black circles). As can be seen in particular in the magnified diagram on the right, the two curves are no longer identical but small changes are indeed induced by the diquark component. In terms of the (extrapolated) real parts of the masses of the resonance, this becomes evident in Table I. Whereas the masses are identical (within error) in the two setups with four-quark contributions only,
$M=416(26) \mathrm{MeV},{ }^{1}$ there are small corrections of the order of $3 \%$ once the mixing with the quarkonium component is taken into account: $M=472 \pm 22$ vs $M=456 \pm 24 \mathrm{MeV}$. Thus, the overlap of the diquark components with the $q \bar{q}$ components (and therefore, their indirect effect on the mass) is larger than their overlap with the $\pi \pi$ components. We do not have a deep explanation why this is the case. Anyway, their contributions remain very small.

It is also interesting to note that the eigenvalue curve of the full system follows the corresponding one for the quarkonium system (blue stars) for spacelike values of $P^{2}=-M^{2}$ and only deviates shortly before we cannot follow the curve any more. By comparison with the curve without quarkonium (violet crosses), it seems clear that this is the region where the two-pion component becomes important and begins to dominate the curve. This ties in with fact that this is precisely the region where the pion poles enter the integration region of the system of BSEs. By comparing the results of the extrapolation ( $456 \pm 24$ vs $416 \pm 26$ ), we find that although the quarkonium contribution is subdominant, it shifts the resulting mass of the resonance by almost $10 \%$, which is quantitatively non-negligible.

Nevertheless, the main conclusion of Ref. [17] remains intact and is in fact reaffirmed: the physical state is dominated by $\pi \pi$ components. Thus it must have a large decay width into two pions. Furthermore, its mass,

$$
M_{0^{++}}=456 \pm 24 \mathrm{MeV}
$$

is in the right ballpark to make the identification with the $f_{0}(500)$ extracted from pion scattering experiments using

\footnotetext{
${ }^{1}$ The small difference of this result with the one reported in Ref. [17] is due to improved numerics and extrapolation procedures.
} 
TABLE I. The masses of different setups isolating and mixing four-quark components and quarkonia from and with each other. The error estimates stem from variations of the curve extrapolations using different rational functions.

\begin{tabular}{lcc}
\hline \hline$[\mathrm{MeV}]$ & Ground state mass & First excitation \\
\hline$\pi \pi$ & $416 \pm 26$ & $970 \pm 130$ \\
$\pi \pi+0^{+} 0^{+}$ & $416 \pm 26$ & $970 \pm 130$ \\
$q \bar{q}$ & $667 \pm 2$ & $1036 \pm 8$ \\
$\pi \pi+q \bar{q}$ & $472 \pm 22$ & $1080 \pm 280$ \\
$\pi \pi+0^{+} 0^{+}+q \bar{q}$ & $456 \pm 24$ & $1110 \pm 110$ \\
\hline \hline
\end{tabular}

dispersion theory at $M=\left(449_{-16}^{+22}\right)-i(275 \pm 12) \mathrm{MeV}$ [5] very plausible.

It is interesting to compare the results for the first radial excitation in all setups considered so far, given in the third column of Table I. In all combinations, we do find a first radial excitation close to or even above one GeV. Most results have large error bars due to the considerable distance of extrapolation involved from the region where we are able to determine the eigenvalues to the region, where they cross $\lambda=1$. This together with the fact that all excited states are close together makes a more detailed analysis in analogy to the one discussed above impossible. Most importantly, we cannot say whether any component dominates the first excited state of the full coupled system of BSEs. Nevertheless, it is interesting to speculate what could happen if we augmented the rainbow-ladder twobody kernel $K^{(2)}$ with corrections beyond rainbow ladder. It is well known from BSE calculations of the $q \bar{q}$ system that the pion, protected by chiral symmetry, does not receive any corrections beyond rainbow-ladder. On the other hand, the scalar pure $q \bar{q}$ state receives large additive corrections beyond rainbow ladder of the order of $400 \mathrm{MeV}$ [28-30]. It is therefore plausible, that in the coupled system the ground state dominated by the $\pi \pi$ component will not change by much beyond rainbow ladder. On the other hand, the first radial excitation dominated by the $q \bar{q}$ component will be substantially affected and thus may end up in the region of $1400 \mathrm{MeV}$. Thus, the identification of our first radial excitation with the physical state $f_{0}(1370)$ seems plausible.

\section{B. The decay width of the scalar}

As discussed in Sec. III B, we are not yet in a position to extract a width from the coupled system of equations Fig. 2 but need to resort the simpler $\bar{q} q$ BSE for the scalar state displayed in Fig. 3. Our results for the resonance location from this scalar-meson BSE are shown in the right diagram of Fig. 7, whereas in the left diagram, we give corresponding results for the vector meson for comparison. The boxes mark the regions where data for the analytic continuation were taken from, which generate as output the corresponding locations of the resonance singularities marked by the ellipses.
Let us first concentrate on the left diagram. We clearly see that the error is very large when data are taken from region 1 below the two-pion threshold (indicated by the dotted line). This large error bar makes it virtually impossible to obtain even qualitative results from the analytic continuation in this case. This is the reason why we cannot apply the analytic continuation procedure to the coupled system of two- and four-quark contributions discussed in the previous section, since for that system we only have data available below the threshold. For the two-quark approach of Sec. III B, however, this is possible due to the path deformation described above. We are then able to access the regions 2 and 3, which lead to the very small ellipses shown in the plot. Our results,

$$
M_{\rho}=638(2) \mathrm{MeV}, \quad \Gamma_{\rho}=108(4) \mathrm{MeV},
$$

are in good agreement with the one obtained in [31] using similar methods. Compared to the experimental values, our mass is too small by about $130 \mathrm{MeV}$ and the width by about $40 \mathrm{MeV}$. This does not come completely as a surprise since it is known that beyond rainbow-ladder corrections due to the gluon self-interactions add significantly to the mass of the vector [29]. Whether further corrections are also able to account for the missing width remains to be studied in the future.

The width extracted for the scalar $\sigma$ meson is displayed in the right diagram of Fig. 7. Again, the shaded region on the right is the selected region, where we calculate data from the BSE on the first Riemann sheet. Ellipses on the left stand for the results in the second sheet obtained from our analytic continuation procedure, where errors are proportional to the size of the ellipses. Comparing with the vector case, we observe a significantly larger width. Correspondingly, the errors are also larger. This is to be expected, since the extrapolation needs to probe further into the second Riemann sheet. The results are fairly stable with respect to different choices of input regions; however, regions below threshold do not work well at all, similar to the vector case. The final result is obtained by performing 30 runs, each one with a fixed number of input points within the region $M \in[0.45,0.68] \mathrm{GeV}$ and $\Gamma \in[0.1,0.2] \mathrm{GeV}$. We do this 10 times and obtain a $\sigma$ mass and an error in the form of a standard deviation each time. We then take the average of the ten resulting $\sigma$ masses and calculate the final error using standard error propagation. We obtain

$$
M_{\sigma}=587(12) \mathrm{MeV}, \quad \Gamma_{\sigma}=186(24) \mathrm{MeV} .
$$

It is interesting to compare our results against the calculations from $\pi \pi$ scattering in rainbow ladder from [27]. The (real part of the) masses are (almost) identical to our results. Furthermore, a width was calculated in $[27,50]$ in the impulse approximation, i.e., using pure rainbowladder Bethe-Salpeter amplitudes inside triangle decay 

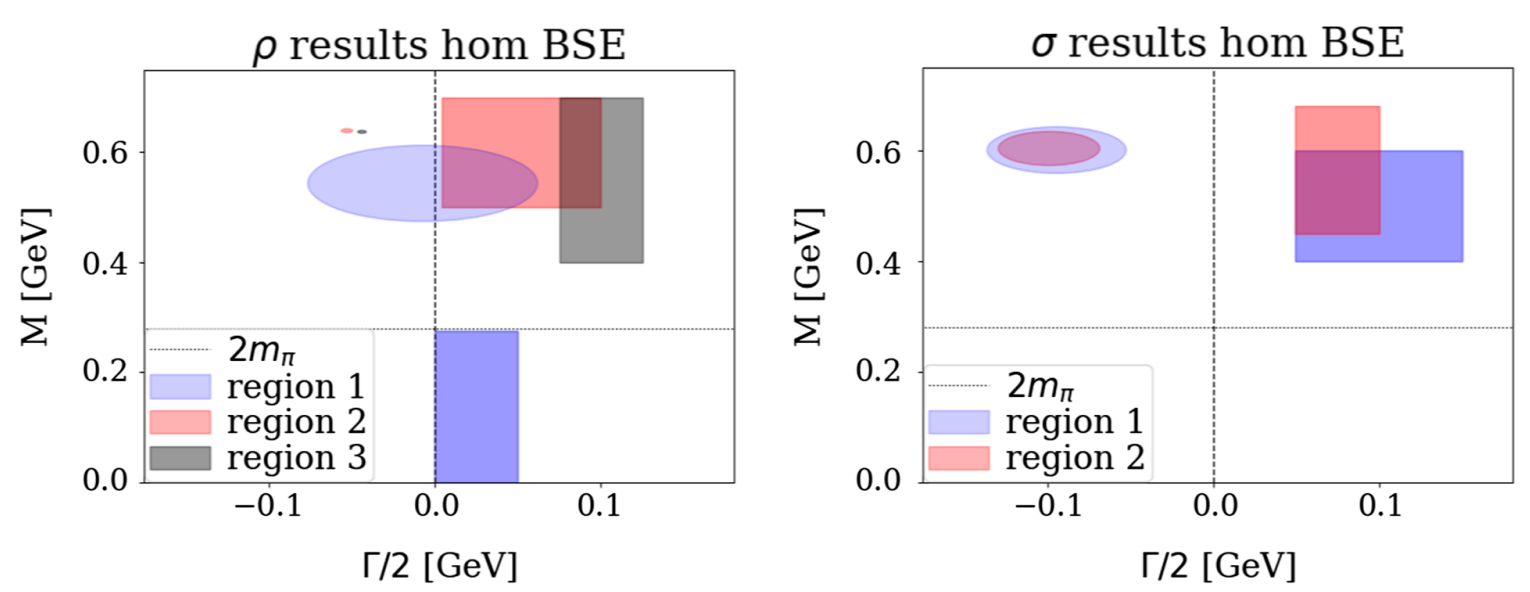

FIG. 7. Location of the complex masses of the $\rho$ (left diagram) and the $\sigma$ meson (right diagram). Both plots show the $\Gamma<0$ half plane of the second Riemann sheet on the left and the $\Gamma>0$ half plane in the first Riemann sheet on the right. Boxes on the right of each plot stand for data calculated from the BSE on the first Riemann sheet for $\Gamma>0$; circles on the left are the results obtained from analytic continuation to the second Riemann sheet for $\Gamma<0$.

diagrams. The resulting widths are similar to the ones we extracted from the second Riemann sheet.

As already discussed above, our result is rather qualitative than quantitative. The real part of the mass is too large, while the width is too small by more than a factor of 2. Of course, these two findings may very well be related to each other, and we therefore may hope that solving the full coupled system of equations, Fig. 2 , in the complex $P^{2}$ plane may yield much more quantitative results for the width. Since the numerical effort involved is considerable, this is left for future work.

\section{SUMMARY}

In this work, we have addressed the mass and the width of the lowest-lying scalar meson in a framework that uses functional methods, i.e., the Dyson-Schwinger and BetheSalpeter equations of QCD. We have solved a coupled system of BSEs that combines contributions from fourquark components (in the form of $\pi \pi$ and diquarkantidiquark amplitudes) and quark-antiquark components. We emphasize that this counting of components is not a counting with respect to Fock states, but that both the fourquark and two-quark contributions each summarize contributions from infinitely many quarks, antiquarks, and gluons. What distinguishes the four- and two-quark contributions instead is the associated valence-quark content needed to build the quantum numbers of the scalar. Our results confirm previous findings from Ref. [17].
The physical state is dominated by the $\pi \pi$ component and the diquark-antidiquark component is almost negligible. Subleading contributions of the order of $10 \%$ of the total mass stem from the quark-antiquark component of the Bethe-Salpeter wave function. This finding explains the small mass of the state, since it is dominated by pseudoGoldstone bosons, and its large decay width into two pions. We have confirmed the latter property (in a simplified system) explicitly by analytically continuing to the second Riemann sheet and determining the associated singularity in the complex energy plane. Our results thus suggest an identification with the experimental $\sigma$ meson, the $f_{0}(500)$. Moreover, we found a first radial excitation in the coupled system of BSEs, which may be identified with the $f_{0}(1370)$, although with much larger uncertainty than is the case for the $\sigma$ meson.

\section{ACKNOWLEDGMENTS}

We are grateful to Sasa Prelovsek, George Rupp, and Marc Wagner for discussions. We are also grateful to the organizers of the very interesting conference "Excited QCD 2020, February 2-8 Krynica Zdroj, Poland," where the idea for part of this work was born. This work was supported by the DFG Grant No. FI 970/11-1, by the Helmholtz International Center for FAIR within the LOEWE program of the State of Hesse, by the Helmholtz Research Academy Hesse for FAIR (HFHF), and by the FCT Investigator Grant No. IF/00898/2015. 
[1] I. Caprini, G. Colangelo, and H. Leutwyler, Phys. Rev. Lett. 96, 132001 (2006).

[2] F. Yndurain, R. Garcia-Martin, and J. Pelaez, Phys. Rev. D 76, 074034 (2007).

[3] R. Garcia-Martin, R. Kaminski, J. Pelaez, and J. Ruiz de Elvira, Phys. Rev. Lett. 107, 072001 (2011).

[4] B. Moussallam, Eur. Phys. J. C 71, 1814 (2011).

[5] J. Pelaez, Phys. Rep. 658, 1 (2016).

[6] R. L. Jaffe, Phys. Rev. D 15, 267 (1977).

[7] N. Achasov and V. Ivanchenko, Nucl. Phys. B315, 465 (1989).

[8] D. Black, A. H. Fariborz, F. Sannino, and J. Schechter, Phys. Rev. D 59, 074026 (1999).

[9] L. Maiani, F. Piccinini, A. Polosa, and V. Riquer, Phys. Rev. Lett. 93, 212002 (2004).

[10] F. Giacosa, Phys. Rev. D 74, 014028 (2006).

[11] E. Klempt and A. Zaitsev, Phys. Rep. 454, 1 (2007).

[12] D. Ebert, R. Faustov, and V. Galkin, Eur. Phys. J. C 60, 273 (2009).

[13] M. Alford and R. Jaffe, AIP Conf. Proc. 688, 208 (2003).

[14] N. Mathur, A. Alexandru, Y. Chen, S. Dong, T. Draper, I. Horvath, F. Lee, K. Liu, S. Tamhankar, and J. Zhang, Phys. Rev. D 76, 114505 (2007).

[15] S. Prelovsek, Acta Phys. Pol. B Proc. Suppl. 3, 975 (2010).

[16] S. Prelovsek, T. Draper, C. B. Lang, M. Limmer, K.-F. Liu, N. Mathur, and D. Mohler, Phys. Rev. D 82, 094507 (2010).

[17] W. Heupel, G. Eichmann, and C. S. Fischer, Phys. Lett. B 718, 545 (2012).

[18] G. Eichmann, C. S. Fischer, and W. Heupel, Phys. Lett. B 753, 282 (2016).

[19] F. E. Close and N. A. Tornqvist, J. Phys. G 28, R249 (2002).

[20] F. Giacosa, Phys. Rev. D 75, 054007 (2007).

[21] G. 't Hooft, G. Isidori, L. Maiani, A. D. Polosa, and V. Riquer, Phys. Lett. B 662, 424 (2008).

[22] J. Londergan, J. Nebreda, J. Pelaez, and A. Szczepaniak, Phys. Lett. B 729, 9 (2014).

[23] J. Pelaez and G. Rios, Phys. Rev. Lett. 97, 242002 (2006).

[24] J. Nieves, A. Pich, and E. Ruiz Arriola, Phys. Rev. D 84, 096002 (2011).

[25] J. Ruiz de Elvira, J. Pelaez, M. Pennington, and D. Wilson, Phys. Rev. D 84, 096006 (2011).

[26] S. Prelovsek and L. Leskovec, Phys. Rev. Lett. 111, 192001 (2013).

[27] S. R. Cotanch and P. Maris, Phys. Rev. D 66, 116010 (2002).
[28] L. Chang and C. D. Roberts, Phys. Rev. Lett. 103, 081601 (2009).

[29] C. S. Fischer and R. Williams, Phys. Rev. Lett. 103, 122001 (2009).

[30] R. Williams, C. S. Fischer, and W. Heupel, Phys. Rev. D 93, 034026 (2016).

[31] R. Williams, Phys. Lett. B 798, 134943 (2019).

[32] A. M. Khvedelidze and A. N. Kvinikhidze, Theor. Math. Phys. 90, 62 (1992).

[33] G. Eichmann, C. S. Fischer, and H. Sanchis-Alepuz, Phys. Rev. D 94, 094033 (2016).

[34] G. Eichmann, H. Sanchis-Alepuz, R. Williams, R. Alkofer, and C. S. Fischer, Prog. Part. Nucl. Phys. 91, 1 (2016).

[35] H. Sanchis-Alepuz and R. Williams, Comput. Phys. Commun. 232, 1 (2018).

[36] S. Yokojima, M. Komachiya, and R. Fukuda, Nucl. Phys. B390, 319 (1993).

[37] S. Capstick and W. Roberts, Prog. Part. Nucl. Phys. 45, S241 (2000).

[38] A. Kvinikhidze and B. Blankleider, Phys. Rev. D 90, 045042 (2014).

[39] P. Maris and C. D. Roberts, Int. J. Mod. Phys. E 12, 297 (2003).

[40] P. C. Wallbott, G. Eichmann, and C. S. Fischer, Phys. Rev. D 100, 014033 (2019).

[41] P. C. Wallbott, G. Eichmann, and C. S. Fischer, arXiv:2003 .12407.

[42] P. Maris and P.C. Tandy, Phys. Rev. C 60, 055214 (1999).

[43] P. Watson, W. Cassing, and P. Tandy, Few Body Syst. 35, 129 (2004).

[44] P. Watson and W. Cassing, Few Body Syst. 35, 99 (2004).

[45] A. S. Miramontes and H. Sanchis-Alepuz, Eur. Phys. J. A 55, 170 (2019).

[46] R.-A. Tripolt, I. Haritan, J. Wambach, and N. Moiseyev, Phys. Lett. B 774, 411 (2017).

[47] R.-A. Tripolt, P. Gubler, M. Ulybyshev, and L. Von Smekal, Comput. Phys. Commun. 237, 129 (2019).

[48] D. Binosi and R.-A. Tripolt, Phys. Lett. B 801, 135171 (2020).

[49] G. Eichmann, P. Duarte, M. Peña, and A. Stadler, Phys. Rev. D 100, 094001 (2019).

[50] V. Mader, G. Eichmann, M. Blank, and A. Krassnigg, Phys. Rev. D 84, 034012 (2011). 